\title{
RC-positive metrics on rationally connected manifolds
}

\author{
Xiaokui Yang \\ Department of Mathematics and Yau Mathematical Sciences Center, Tsinghua University, Beijing 100084, China; \\ E-mail: xkyang@mail.tsinghua.edu.cn.
}

Received: 16 January 2020; Revised: 23 July 2020; Accepted: 16 July 2020

2020 Mathematics Subject Classification: 53C55 (primary); 14E08,14F17,32L20 (secondary)

Keywords: rationally connected; RC-positivity; vanishing theorem

\begin{abstract}
In this paper, we prove that if a compact Kähler manifold $X$ has a smooth Hermitian metric $\omega$ such that $\left(T_{X}, \omega\right)$ is uniformly RC-positive, then $X$ is projective and rationally connected. Conversely, we show that, if a projective manifold $X$ is rationally connected, then there exists a uniformly RC-positive complex Finsler metric on $T_{X}$.
\end{abstract}

\section{Contents}

1 Introduction

2 Uniformly RC-positive Hermitian vector bundles over complex manifolds

3 Vanishing theorems and rational connectedness of compact Kähler manifolds

4 RC-positive metrics on rationally connected manifolds

5 Compact Kähler manifolds with non-negative holomorphic sectional curvature

6 RC-positive Finsler vector bundles over complex manifolds

\section{Introduction}

It is well known that positivity notions in differential geometry and algebraic geometry are closely related, and there are many fundamental results demonstrating their equivalences. For instances, Kodaira's embedding theorem asserts that a line bundle $\mathscr{L}$ over a projective manifold is ample if and only if it has a smooth positive metric; Yau's solution to the Calabi conjecture [53] establishes that a projective manifold $X$ is Fano if and only if it has a Kähler metric $\omega$ with $\operatorname{Ric}(\omega)>0$. As analogous to Yau's result, we obtained in [48] the following result, which also confirms a conjecture of S.-T. Yau:

Theorem 1.1 ([48]). A projective manifold $X$ is uniruled if and only if $X$ has a Kähler metric $\omega$ such that $\operatorname{Ric}(\omega)$ has at least one positive eigenvalue everywhere.

Motivated by Yau's theorem and Theorem 1.1, in this paper, we describe the terminology rational connectedness in algebraic geometry by using notions in differential geometry. It is well-known that rational connectedness is an intermediate concept between Fano-ness and uniruledness, i.e. Fano manifolds are rationally connected [7, 25], and rationally connected manifolds are uniruled.

\footnotetext{
(C) The Author(s), 2020. Published by Cambridge University Press. This is an Open Access article, distributed under the terms of the Creative Commons Attribution licence (http://creativecommons.org/licenses/by/4.0/), which permits unrestricted re-use, distribution, and reproduction in any medium, provided the original work is properly cited.
} 
A projective manifold $X$ is called rationally connected if any two points of $X$ can be connected by some rational curves. It is well known that on a rationally connected projective manifold, one has

$$
H^{0}\left(X,\left(T_{X}^{*}\right)^{\otimes m}\right)=0, \quad \text { for every } \quad m \geq 1 .
$$

A long-standing conjecture of D. Mumford says that the converse is also true:

Conjecture. Let $X$ be a projective manifold. If

$$
H^{0}\left(X,\left(T_{X}^{*}\right)^{\otimes m}\right)=0, \text { for all } m \geq 1,
$$

then $X$ is rationally connected.

This conjecture holds when $\operatorname{dim} X \leq 3$ [25], and not much has been known in higher dimensions; and we refer to $[25,7,17,24,19,41,6,9,11,5,8,28,12,49]$, and the references therein.

In this paper, we obtain a differential geometric criterion for rational connectedness. One of the key ingredients is the following geometric concept.

Definition 1.2. A Hermitian holomorphic vector bundle $(\mathscr{E}, h)$ over a complex manifold $X$ is called uniformly $R C$-positive (respectively, semi-positive) if at each point $q \in X$, there exists some nonzero vector $u \in T_{q} X$ such that for every nonzero vector $v \in \mathscr{E}_{q}$,

$$
R^{\mathscr{E}}(u, \bar{u}, v, \bar{v})>0 \quad(\text { respectively, } \geq 0) .
$$

Similarly, $(\mathscr{E}, h)$ is called uniformly $R C$-quasi-positive if it is uniformly RC-semi-positive at all points of $X$ and uniformly RC-positive at some point of $X$.

We establish in Corollary 3.1 that if $(\mathscr{E}, h)$ is a uniformly RC-positive vector bundle over a compact complex manifold $X$, then

$$
H^{0}\left(X,\left(\mathscr{E}^{*}\right)^{\otimes m}\right)=0, \quad \text { for every } m \geq 1
$$

As motivated by this result and Mumford's conjecture, we investigate complex manifolds with uniformly RC-positive tangent bundles. Indeed, there are many such examples. For instance,

○ Kähler manifolds with positive holomorphic sectional curvature (Theorem 5.1),

- Fano manifolds with non-negative holomorphic sectional curvature [34],

○ Hopf manifolds $\mathbb{S}^{1} \times \mathbb{S}^{2 n+1}$ [30, formula (6.4)],

$\circ$ products of complex manifolds with uniformly RC-positive tangent bundles (Proposition 2.15).

Let $X$ be a compact complex manifold. The holomorphic tangent bundle $T_{X}$ is called uniformly RCpositive if it admits a smooth Hermitian metric $\omega$ such that $\left(T_{X}, \omega\right)$ is uniformly RC-positive. The first main result of this paper is a criterion of rational connectedness for compact Kähler manifolds:

Theorem 1.3. Let $X$ be a compact Kähler manifold. If the tangent bundle $T_{X}$ is uniformly RC-positive, then $X$ is projective and rationally connected. In particular, $X$ is simply connected.

We propose in Section 4 a conjecture converse to Theorem 1.3: if $X$ is rationally connected, then $T_{X}$ should be (uniformly) RC-positive. We present there some evidence for this conjecture. Theorem 1.3 also holds if the uniformly RC-positive Hermitian metrics are replaced by uniformly RC-positive Finsler metrics. We refer to Theorem 6.7 for more details.

It is well known that a projective manifold $X$ is rationally connected if and only if it has a very free rational curve $C$, i.e. $\left.T_{X}\right|_{C}$ is ample. Recall that, on a smooth curve $C$, a vector bundle $\mathscr{E}$ is uniformly 
RC-positive if and only if $\mathscr{E}$ is ample. Hence, roughly speaking, the uniform RC-positivity of $T_{X}$ is an analytical analogue of the existence of very free rational curves on $X$ (see also [42, Theorem 6.6]). Before giving a weak converse to Theorem 1.3, we fix the notations. Let $\mathscr{E}$ be a holomorphic vector bundle over $X$ and $\mathbb{P}\left(\mathscr{E}^{*}\right)$ be the projective bundle of $\mathscr{E}$. The tautological line bundle of $\mathbb{P}\left(\mathscr{E}^{*}\right)$ is denoted by $\mathcal{O}_{\mathscr{E}}(1)$. For instance, $\mathscr{E}$ is called ample if $\mathcal{O}_{\mathscr{C}}(1)$ is an ample line bundle. The second main result of this paper is:

Theorem 1.4. A projective manifold $X$ is rationally connected if and only if the line bundle $\mathcal{O}_{\Lambda^{p}} T_{X}^{*}(-1)$ is uniformly RC-positive for every $1 \leq p \leq \operatorname{dim} X$.

It is well known that there exists a one-to-one correspondence between the set of Hermitian metrics on $\mathcal{O}_{\mathscr{E}}(-1)$ and the set of Finsler metrics on $\mathscr{E}^{*}$. Hence, we can deduce that $X$ is rationally connected if and only if $X$ has certain 'RC-positive' Finsler metrics. It would be more satisfactory if the Finsler metrics could be turned into smooth Hermitian metrics. We refer to Section 6 for more details about (uniformly) RC-positive Finsler metrics.

As an application of Theorem 1.3, we obtain the following Liouville type result.

Corollary 1.5. Let $X$ be a compact Kähler manifold. If $T_{X}$ is uniformly RC-positive, then there is no non-constant holomorphic map from $X$ to a compact complex manifold $Y$ without any rational curve.

There are many compact complex manifolds that contain no rational curves: for instance, Kobayashi hyperbolic manifolds, Abelian varieties, and Hopf manifolds. Corollary 1.5 generalizes many classical results in differential geometry (e.g. [54]). Note also that the Kähler condition in Corollary 1.5 is necessary. For instance, Hopf manifolds contain no rational curves, and they have uniformly RC-positive tangent bundles. We refer to $[50,51]$ for analogous results and some conjectures.

The following result demonstrates that we can deform RC-semi-positive metrics:

Theorem 1.6. Let $X$ be a compact Kähler manifold. Suppose there exists a smooth Hermitian metric $h$ on $T_{X}$ such that $\left(T_{X}, h\right)$ is uniformly $R C$-semi-positive over $X$. If $S$ is an open subset of $X$ such that $\left(T_{X}, h\right)$ is uniformly $R C$-positive over $X \backslash S$, and $\bar{S}$ is contained in some strongly pseudoconvex domain of $X$, then $X$ has a uniformly $R C$-positive Hermitian metric $\widetilde{h}$. Moreover, $X$ is projective and rationally connected.

As an application of Theorem 1.6, we obtain

Theorem 1.7. Let $(X, \omega)$ be a compact Kähler manifold with non-negative holomorphic sectional curvature. If $S$ is an open subset of $X$ such that $\left(T_{X}, \omega\right)$ is uniformly $R C$-positive over $X \backslash S$, and $\bar{S}$ is contained in some strongly pseudoconvex domain of $X$, then $T_{X}$ is uniformly RC-positive. Moreover, $X$ is projective and rationally connected.

A special case of Theorem 1.7 is

Corollary 1.8. Let $(X, \omega)$ be a compact Kähler manifold with positive holomorphic sectional curvature. Then $\left(T_{X}, \omega\right)$ is uniformly $R C$-positive. Moreover, $X$ is projective and rationally connected. In particular, $X$ is simply connected.

Corollary 1.8 confirms a well-known conjecture [55, Problem 47] of S.-T. Yau. It was first proved in our previous paper [49, Theorem 1.7]. Recently, Lei Ni and Fangyang Zheng introduced in [38, 39] some interesting curvature notions that can ensure the projectivity of compact Kähler manifolds.

Inspired by the above results, we propose the following conjecture:

Conjecture 1.9. Let $X$ be a compact Kähler manifold. If $X$ has a smooth Hermitian metric $\omega$ with quasi-positive holomorphic sectional curvature (or with uniformly $R C$-quasi-positive $\left(T_{X}, \omega\right)$ ), then $X$ is projective and rationally connected. 
We need to point out that S. Matsumura confirmed in [33] a special case of Conjecture 1.9: if $X$ is a projective manifold and has a Kähler metric with quasi-positive holomorphic sectional curvature, then $X$ is rationally connected (see also an approach in [21]).

The rest of the paper is organized as follows. In Section 2, we introduce the concept of uniform RC-positivity and investigate its geometric properties. In Section 3, we derive vanishing theorems for uniformly RC-positive vector bundles and prove Theorem 1.3 and Theorem 1.6. In Section 4, we prove Theorem 1.4 and propose more questions. In Section 5, we give a proof of Theorem 1.7. In Section 6, we present further generalizations on Finsler metrics.

\section{Uniformly RC-positive Hermitian vector bundles over complex manifolds}

Let $(\mathscr{E}, h)$ be a Hermitian holomorphic vector bundle over a complex manifold $X$ with Chern connection $\nabla$. Let $\left\{z^{i}\right\}_{i=1}^{n}$ be the local holomorphic coordinates on $X$ and $\left\{e_{\alpha}\right\}_{\alpha=1}^{r}$ be a local frame of $\mathscr{E}$. The curvature tensor $R^{\mathscr{E}} \in \Gamma\left(X, \Lambda^{1,1} T_{X}^{*} \otimes \operatorname{End}(\mathscr{E})\right)$ has components

$$
R_{i \bar{j} \alpha \bar{\beta}}^{\mathscr{E}}=-\frac{\partial^{2} h_{\alpha \bar{\beta}}}{\partial z^{i} \partial \bar{z}^{j}}+h^{\gamma \bar{\delta}} \frac{\partial h_{\alpha \bar{\delta}}}{\partial z^{i}} \frac{\partial h_{\gamma \bar{\beta}}}{\partial \bar{z}^{j}} .
$$

(Here and henceforth, we sometimes adopt the Einstein convention for summation.) If $\left(X, \omega_{g}\right)$ is a Hermitian manifold, then $\left(T_{X}, g\right)$ has Chern curvature components

$$
R_{i \bar{j} k \bar{\ell}}=-\frac{\partial^{2} g_{k \bar{\ell}}}{\partial z^{i} \partial \bar{z}^{j}}+g^{p \bar{q}} \frac{\partial g_{k \bar{q}}}{\partial z^{i}} \frac{\partial g_{p \bar{\ell}}}{\partial \bar{z}^{j}} .
$$

The Chern-Ricci curvature $\operatorname{Ric}\left(\omega_{g}\right)$ of $\left(X, \omega_{g}\right)$ is represented by $R_{i \bar{j}}=g^{k \bar{\ell}} R_{i \bar{j} k \bar{\ell}}$, and the second Chern-Ricci curvature $\operatorname{Ric}^{(2)}\left(\omega_{g}\right)$ has components $R_{k \bar{\ell}}^{(2)}=g^{i \bar{j}} R_{i \bar{j} k \bar{\ell}}$.

Definition 2.1. A Hermitian holomorphic vector bundle $(\mathscr{E}, h)$ over a complex manifold $X$ is called Griffiths positive if at each point $q \in X$, and for any nonzero vector $v \in \mathscr{E}_{q}$ and any nonzero vector $u \in T_{q} X$,

$$
R^{\mathscr{E}}(u, \bar{u}, v, \bar{v})>0 .
$$

As analogous to the Griffiths positivity, we introduced in [49] the following concept.

Definition 2.2. A Hermitian holomorphic vector bundle $(\mathscr{E}, h)$ over a complex manifold $X$ is called $R C$-positive at point $q \in X$ if for each nonzero vector $v \in \mathscr{E}_{q}$, there exists some nonzero vector $u \in T_{q} X$ such that

$$
R^{\mathscr{E}}(u, \bar{u}, v, \bar{v})>0
$$

$(\mathscr{E}, h)$ is called $R C$-positive if it is RC-positive at every point of $X$.

Remark 2.3. Similarly, one can define semi-positivity, negativity, etc. A Hermitian line bundle $\left(\mathscr{L}, h^{\mathscr{L}}\right)$ is RC-positive if and only if its curvature $-\sqrt{-1} \partial \bar{\partial} \log h^{\mathscr{L}}$ has at least one positive eigenvalue at each point of $X$.

The following vanishing theorem is one of the key ingredients because it introduces the terminology $R C$-positivity.

Theorem 2.4. Let $X$ be a compact complex manifold. If $(\mathscr{E}, h)$ is $R C$-positive, then

$$
H^{0}\left(X, \mathscr{E}^{*}\right)=0 .
$$


Proof. An algebraic proof is included in [50, Lemma 2.10]. Here we use an alternative proof by a simple maximum principle. Since $(\mathscr{E}, h)$ is RC-positive, the induced bundle $\left(\mathscr{E}^{*}, g\right)$ is RC-negative: i.e. at every point $q$, for any nonzero section $v$ of $\mathscr{E}^{*}$, there exists a nonzero vector $u$ such that

$$
R^{\mathscr{E}^{*}}(u, \bar{u}, v, \bar{v})<0 .
$$

For any $\sigma \in H^{0}\left(X, \mathscr{E}^{*}\right)$, we have

$$
\partial \bar{\partial}|s|_{g}^{2}=\langle\nabla s, \nabla s\rangle_{g}-R^{\mathscr{E}^{*}}(\bullet, \bullet, s, \bar{s}) .
$$

Suppose $|s|_{g}^{2}$ attains its maximum at some point $p$, and $|s|_{g}^{2}(p)>0$. By applying the maximum principle to (2.6), we get a contradiction. Hence, we deduce $s=0$ and $H^{0}\left(X, \mathscr{E}^{*}\right)=0$.

In particular, we obtain a simple criterion for the projectivity of compact Kähler manifolds.

Corollary 2.5. Let $X$ be a compact Kähler manifold. Suppose $\Lambda^{2} T_{X}$ is RC-positive; then $X$ is projective. Proof. By Theorem 2.4, we have $H_{\bar{\partial}}^{2,0}(X)=H_{\bar{\partial}}^{0,2}(X)=0$. Hence, by the Kodaira theorem ([23, Theorem 1]; see also [22, Proposition 3.3.2 and Corollary 5.3.3]), the Kähler manifold $X$ is projective.

We introduce a notion slightly stronger than RC-positivity.

Definition 2.6. A Hermitian holomorphic vector bundle $(\mathscr{E}, h)$ over a complex manifold $X$ is called uniformly $R C$-positive at point $q \in X$ if there exists some vector $u \in T_{q} X$ such that for any nonzero vector $v \in \mathscr{E}_{q}$, one has

$$
R^{\mathscr{E}}(u, \bar{u}, v, \bar{v})>0
$$

$(\mathscr{E}, h)$ is called uniformly $R C$-positive if it is uniformly RC-positive at every point $q \in X$.

Remark 2.7. We can define uniform RC-negativity (and, respectively, uniform RC-non-negativity, uniform RC-non-positivity) in a similar way. We can also define uniformly $R C$-positivity along $k$ linearly independent directions if there exist $k$ linearly independent vectors $u_{1}, \cdots, u_{k} \in T_{q} X$ such that for any nonzero vector $v \in \mathscr{E}_{q}$ and for each $i=1, \cdots, k$, one has

$$
R^{\mathscr{E}}\left(u_{i}, \bar{u}_{i}, v, \bar{v}\right)>0 .
$$

Remark 2.8. It is easy to see that, for a line bundle $(\mathscr{L}, h)$, RC-positivity and uniform RC-positivity are actually equivalent.

Proposition 2.9. Let $(\mathscr{E}, h)$ be a Hermitian holomorphic vector bundle over a compact complex manifold $X$. Then the following statements are equivalent:

1. $(\mathscr{E}, h)$ is uniformly $R C$-positive.

2. For any Hermitian metric $\omega$ on $X$, there exists a positive constant $C=C(\omega, h)$ such that for any point $q \in X$, there exists a unit vector $u \in T_{q} X$ such that

$$
R^{\mathscr{E}}(u, \bar{u}, v, \bar{v}) \geq C|v|_{h}^{2}, \quad \text { for every } \quad v \in \mathscr{E}_{q} .
$$

Proof. (2) $\Longrightarrow(1)$ is obvious. For (2) $\Longrightarrow(1)$, let

$$
C=\inf _{q \in X} \sup _{u \in T_{q} X \backslash\{0\}} \inf _{v \in \mathscr{C}_{q} \backslash\{0\}} \frac{R^{\mathscr{E}}(u, \bar{u}, v, \bar{v})}{|u|_{\omega}^{2}|v|_{h}^{2}} .
$$


We claim $C>0$. Indeed, if $C \leq 0$, by compactness of $X$, there exists some $q \in X$ such that

$$
\sup _{u \in T_{q} X \backslash\{0\}} \inf _{v \in \mathscr{E}_{q} \backslash\{0\}} \frac{R^{\mathscr{E}}(u, \bar{u}, v, \bar{v})}{|u|_{\omega}^{2}|v|_{h}^{2}} \leq 0 .
$$

Hence, for any unit vector $u \in T_{q} X$,

$$
\inf _{v \in \mathscr{C}_{q} \backslash\{0\}} \frac{R^{\mathscr{E}}(u, \bar{u}, v, \bar{v})}{|v|_{h}^{2}} \leq 0,
$$

which contradicts the fact that $(\mathscr{E}, h)$ is uniformly RC-positive at point $q \in X$. It is obvious that (2.8) follows from the definition (2.9) of the constant $C$.

Proposition 2.10. If $\left(\mathscr{E}, h_{1}\right)$ is uniformly $R C$-positive and $\left(\mathscr{F}, h_{2}\right)$ is Griffiths semi-positive, then $(\mathscr{E} \otimes$ $\left.\mathscr{F}, h_{1} \otimes h_{2}\right)$ is uniformly RC-positive.

Proof. It follows from the curvature formula $R^{\mathscr{E} \otimes \mathscr{F}}=R^{\mathscr{E}} \otimes \operatorname{Id}_{\mathscr{F}}+\operatorname{Id}_{\mathscr{E}} \otimes R^{\mathscr{F}}$.

The following results can be deduced by similar methods, as in [49, Theorem 3.5].

Corollary 2.11. Let $(\mathscr{E}, h)$ be a Hermitian vector bundle over a compact complex manifold X.

1. If $(\mathscr{E}, h)$ is uniformly $R C$-positive, then $(\mathscr{E}, h)$ is $R C$-positive.

2. $(\mathscr{E}, h)$ is uniformly $R C$-positive if and only if $\left(\mathscr{E}^{*}, h^{*}\right)$ is uniformly $R C$-negative.

3. If $(\mathscr{E}, h)$ is uniformly $R C$-negative, every sub-bundle $\mathcal{S}$ of $\mathscr{E}$ is uniformly $R C$-negative.

4. If $(\mathscr{E}, h)$ is uniformly $R C$-positive, every quotient bundle $\mathbb{Q}$ of $\mathscr{E}$ is uniformly $R C$-positive.

5. If $(\mathscr{E}, h)$ is uniformly $R C$-positive, every line sub-bundle $\mathscr{L}$ of $\mathscr{E}^{*}$ is not pseudo-effective.

Uniform RC-positivity has some important functorial properties, which are not clearly satisfied by $R C$ positivity.

Proposition 2.12. If $(\mathscr{E}, h)$ is uniformly $R C$-positive, then $\left(\mathscr{E}^{\otimes m}, h^{\otimes m}\right)$ are uniformly $R C$-positive for $m \in \mathbb{N}^{+}$. Similarly, $\operatorname{Sym}^{\otimes k} \mathscr{E}\left(k \in \mathbb{N}^{+}\right)$and $\Lambda^{p \mathscr{E}}(1 \leq p \leq \operatorname{rk}(\mathscr{E}))$ are all uniformly $R C$-positive.

Proof. Fix a smooth metric on the compact complex manifold $X$. For any $q \in X$, we choose a unit vector $u \in T_{q} X$ such that

$$
\inf _{v \in \mathscr{E}_{q} \backslash\{0\}} \frac{R^{\mathscr{E}}(u, \bar{u}, v, \bar{v})}{|v|_{h}^{2}} \geq C>0 .
$$

Let $\left\{e_{1}, \cdots, e_{r}\right\}$ be a local unitary frame of $\mathscr{E}$ at point $q$ with respect to $h$. Then for any local vector $v=\sum_{i_{1}, \cdots, i_{m}} v^{i_{1} \cdots i_{m}} e_{i_{1}} \otimes \cdots \otimes e_{i_{m}} \in \Gamma\left(X, \mathscr{E}{ }^{\otimes m}\right)$, by using the tensor product curvature formula of $\left(\mathscr{E}^{\otimes m}, h^{\otimes m}\right)$, we have

$$
\begin{aligned}
R^{\mathscr{E} \otimes m}(u, \bar{u}, v, \bar{v})= & \sum_{i_{2}, \cdots, i_{m}} R^{\mathscr{E}}\left(u, \bar{u}, \sum_{i_{1}} v^{i_{1} i_{2} \cdots i_{m}} e_{i_{1}}, \overline{\sum_{j_{1}} v^{j_{1} i_{2} \cdots i_{m}} e_{j_{1}}}\right)+\cdots \\
& +\sum_{i_{1}, i_{2}, \cdots, i_{m-1}} R^{\mathscr{E}}\left(u, \bar{u}, \sum_{i_{m}} v^{i_{1} \cdots i_{m-1} i_{m}} e_{i_{m}}, \sum_{j_{m}} \overline{v^{i_{1} \cdots i_{m-1} j_{m}} e_{j_{m}}}\right) \\
\geq & C\left(\sum_{i_{2}, \cdots, i_{m}} \sum_{i_{1}}\left|v^{i_{1} i_{2} \cdots i_{m}}\right|^{2}+\cdots+\sum_{i_{1}, \cdots, i_{m-1}} \sum_{i_{m}}\left|v^{i_{1} i_{2} \cdots i_{m}}\right|^{2}\right) \\
\geq & m C|v|^{2},
\end{aligned}
$$


where the first inequality follows from (2.10). Hence $\left(\mathscr{E}{ }^{\otimes m}, h^{\otimes m}\right)$ is uniformly RC-positive. Similarly, we can show $\operatorname{Sym}^{\otimes k} \mathscr{E}\left(k \in \mathbb{N}^{+}\right)$and $\Lambda^{p \mathscr{E}}(1 \leq p \leq \operatorname{rk}(\mathscr{E}))$ are all uniformly RC-positive.

Remark 2.13. It is not hard to see that all Schur powers of a uniformly RC-positive vector bundle are uniformly RC-positive.

It is easy to see that if two vector bundles $\mathscr{E}$ and $\mathscr{F}$ are uniformly RC-positive, their tensor product is not necessarily uniformly RC-positive.

Example 2.14. Let $X=\mathbb{P}^{1} \times C$, where $C$ is a Riemann surface of genus $g \geq 2$. It is easy to see that both $\mathscr{L}_{1}=K_{X}$ and $\mathscr{L}_{2}=K_{X}^{-1}$ are uniformly RC-positive with respect to metric induced by the canonical metric on $X$. However, their tensor product $\mathscr{L}_{1} \otimes \mathscr{L}_{2}$ is a trivial line bundle that cannot be uniformly RC-positive.

However, we have the following property.

Proposition 2.15. Let $\left(X, \omega_{X}\right)$ and $\left(Y, \omega_{Y}\right)$ be two Hermitian manifolds. Suppose both $\left(T_{X}, \omega_{X}\right)$ and $\left(T_{Y}, \omega_{Y}\right)$ are uniformly $R C$-positive; then $\left(T_{X} \otimes T_{Y}, \omega_{X} \otimes \omega_{Y}\right)$ is also uniformly RC-positive over $X \times Y$.

Proof. For simplicity, we do not use pullback notions. Since both $\left(T_{X}, \omega_{X}\right)$ and $\left(T_{Y}, \omega_{Y}\right)$ are uniformly RC-positive, for any point $p \in X$ and $q \in Y$, there exist vectors $u_{X} \in T_{p} X$ and $u_{Y} \in T_{q} Y$ such that

$$
R^{\left(T_{X}, \omega_{X}\right)}\left(u_{X}, \bar{u}_{X}, v, v\right)>0 \quad \text { for any } \quad v \in T_{p} X
$$

and

$$
R^{\left(T_{Y}, \omega_{Y}\right)}\left(u_{Y}, \bar{u}_{Y}, w, w\right)>0 \text { for any } \quad w \in T_{q} Y
$$

The curvature tensor of $\left(T_{X} \otimes T_{Y}, \omega_{X} \otimes \omega_{Y}\right)$ is given by

$$
R^{\left(T_{X} \otimes T_{Y}, \omega_{X} \otimes \omega_{Y}\right)}=R^{\left(T_{X}, \omega_{X}\right)} \otimes \operatorname{Id}_{Y}+\operatorname{Id}_{X} \otimes R^{\left(T_{Y}, \omega_{Y}\right)} .
$$

Given any point $(p, q) \in X \times Y$, there exists a vector $u_{X} \otimes u_{Y} \in T_{p} X \otimes T_{q} Y$ such that for any $v=\sum v_{i} \otimes w_{i} \in T_{p} X \otimes T_{q} Y:$

$$
\begin{aligned}
& R^{\left(T_{X} \otimes T_{Y}, \omega_{X} \otimes \omega_{Y}\right)}\left(u_{X} \otimes u_{Y}, \bar{u}_{X} \otimes \bar{u}_{Y}, v, \bar{v}\right) \\
= & \sum R^{\left(T_{X}, \omega_{X}\right)}\left(u_{X}, \bar{u}_{X}, v_{i}, v_{i}\right)\left|w_{i}\right|^{2}+\sum R^{\left(T_{Y}, \omega_{Y}\right)}\left(u_{Y}, \bar{u}_{Y}, w_{i}, w_{i}\right)\left|v_{i}\right|^{2}>0 .
\end{aligned}
$$

Hence, $\left(T_{X} \otimes T_{Y}, \omega_{X} \otimes \omega_{Y}\right)$ is uniformly RC-positive over $X \times Y$.

\section{Vanishing theorems and rational connectedness of compact Kähler manifolds}

In this section, we derive vanishing theorems for uniformly RC-positive vector bundles and prove Theorem 1.3 and Theorem 1.6.

Corollary 3.1. If $(\mathscr{E}, h)$ is a uniformly $R C$-positive vector bundle over a compact complex manifold $X$, then

$$
H^{0}\left(X,\left(\mathscr{E}^{*}\right)^{\otimes m}\right)=0, \quad \text { for every } \quad m \geq 1 .
$$

Proof. It follows from Proposition 2.12, Corollary 2.11, and Theorem 2.4.

The following special case is of particular interest. 
Corollary 3.2. Let $X$ be a compact complex manifold. If there exists a smooth Hermitian metric $h$ such that $\left(T_{X}, h\right)$ is uniformly $R C$-positive, then $H_{\bar{\partial}}^{p, 0}(X)=0$ for $1 \leq p \leq \operatorname{dim} X$ and

$$
H^{0}\left(X,\left(T_{X}^{*}\right)^{\otimes m}\right)=0, \text { for all } m \geq 1 .
$$

Proof. By Corollary 2.11 and Proposition 2.12, we know $\Lambda^{p} T_{X}$ and $T_{X}^{\otimes m}$ are all uniformly RC-positive. Hence by Corollary $3.1, H_{\bar{\partial}}^{p, 0}(X) \cong H^{0}\left(X, \Lambda^{p} T_{X}^{*}\right)=0$.

Theorem 3.3. Let $(\mathscr{E}, h)$ be a uniformly $R C$-positive vector bundle over a compact complex manifold $X$. Then for any line bundle $\mathscr{L}$ over $X$, there exists a positive constant $C=C(\mathscr{L})$ such that $\mathscr{E}^{\otimes m} \otimes \mathscr{L}^{\otimes k}$ is uniformly $R C$-positive for all $m, k \in \mathbb{N}^{+}$with $m \geq C k$. In particular,

$$
H^{0}\left(X,\left(\mathscr{E}^{*}\right)^{\otimes m} \otimes\left(\mathscr{L}^{*}\right)^{\otimes k}\right)=0 .
$$

Proof. We fix an arbitrary smooth Hermitian metric $g$ on $\mathscr{L}$ and assume that the curvature of $(\mathscr{L}, g)$ is bounded from below by a negative constant $-B$. Fix a point $q \in X$. Since $(\mathscr{E}, h)$ is uniformly RCpositive, there exist a positive constant $C$ and a unit vector $u \in T_{q} X$ such that

$$
\inf _{v \in \mathscr{E}_{q} \backslash\{0\}} \frac{R^{\mathscr{E}}(u, \bar{u}, v, \bar{v})}{|v|_{h}^{2}} \geq C>0 .
$$

By a similar computation as in Proposition 2.12, for any $v \in \mathscr{E}_{q}^{\otimes m}$, we have

$$
R^{\left(\mathscr{C}^{\otimes m}, h^{\otimes m}\right)}(u, \bar{u}, v, \bar{v}) \geq m C|v|^{2} .
$$

We choose a local unitary frame $e \in \Gamma(X, \mathscr{L})$ centered at $q$; then

$$
\begin{aligned}
& R^{\mathscr{E}^{\otimes m} \otimes \mathscr{L}^{\otimes k}}\left(u, \bar{u}, v \otimes e^{\otimes k}, \overline{v \otimes e^{\otimes k}}\right)=R^{\left(\mathscr{C}^{\otimes m}, h^{\otimes m}\right)}(u, \bar{u}, v, \bar{v})+k|v|^{2} R^{\mathscr{L}}(u, \bar{u}) \\
& \geq m C|v|^{2}-k B|v|^{2} \text {. }
\end{aligned}
$$

Hence if $m \geq k B / C+1$, then

$$
R^{\mathscr{C} \otimes m} \otimes \mathscr{L}^{\otimes k}\left(u, \bar{u}, v \otimes e^{\otimes k}, \overline{v \otimes e^{\otimes k}}\right) \geq|v|^{2} .
$$

Hence, $\mathscr{E}^{\otimes m} \otimes \mathscr{L}^{\otimes k}$ is uniformly RC-positive. By Corollary 3.1, we obtain (3.3).

Recall that $\left\{z^{i}\right\},\left\{e_{\alpha}\right\}$ are the local holomorphic coordinates and holomorphic frames on $X$ and $\mathscr{E}$, respectively.

Lemma 3.4. Let $\widetilde{h}=e^{-f} h$ for some $f \in C^{2}(X, \mathbb{R})$. Then the curvature tensor $\widetilde{R}$ of $(\mathscr{E}, \widetilde{h})$ has the expression

$$
\widetilde{R}_{i \bar{j} \alpha \bar{\beta}}=e^{-f}\left(R_{i \bar{j} \alpha \bar{\beta}}+f_{i \bar{j}} h_{\alpha \bar{\beta}}\right),
$$

where $R$ is the curvature tensor of $(\mathscr{E}, h)$.

Proof. It follows by a straightforward computation.

Theorem 3.5. Let $(\mathscr{E}, h)$ be a Hermitian holomorphic vector bundle over a compact complex manifold $X$. Suppose $(\mathscr{E}, h)$ is uniformly $R C$-semi-positive over $X$. If there exist two open subsets $S$ and $U$ of $X$ such that

1. $U$ is strongly pseudoconvex and $\bar{S} \subset U$

2. $(\mathscr{E}, h)$ is uniformly $R C$-positive on $X \backslash S$

then there exists a smooth Hermitian metric $\widetilde{h}$ on $\mathscr{E}$ such that $(\mathscr{E}, \widetilde{h})$ is uniformly $R C$-positive over $X$. 
Proof. Fix an arbitrary smooth Hermitian metric $\omega$ on $X$. We define

$$
C=\inf _{q \in X \backslash S} \sup _{u \in T_{q} X \backslash\{0\}} \inf _{v \in \mathscr{E}_{q} \backslash\{0\}} \frac{R(u, \bar{u}, v, \bar{v})}{|u|_{\omega}^{2}|v|_{h}^{2}} .
$$

Since $X \backslash S$ is compact and $(\mathscr{E}, h)$ is uniformly RC-positive over $X \backslash S$, it is easy to see that $C>0$. Hence, we conclude that there exists a 'cut-off' function $f \in C^{\infty}(X, \mathbb{R})$ such that

1. Over $X$, we have

$$
(\sqrt{-1} \partial \bar{\partial} f)(u, \bar{u}) \geq-\frac{C}{2}|u|_{\omega}^{2} .
$$

2. Over $\bar{S}$, we have

$$
(\sqrt{-1} \partial \bar{\partial} f)(u, \bar{u}) \geq C_{1}|u|_{\omega}^{2}
$$

for some positive constant $C_{1}$.

Indeed, since $U$ is strongly pseudoconvex, there exists a smooth strictly plurisubharmonic function $\varphi \in \operatorname{Psh}(U)$. In particular, there exists a positive constant $\widetilde{C}_{1}$ such that $(\sqrt{-1} \partial \bar{\partial} \varphi)(u, \bar{u}) \geq \widetilde{C}_{1}|u|_{\omega}^{2}$ over the compact set $\bar{S}$. Next, we can extend the smooth function $\left.\varphi\right|_{\bar{S}}$ to $X$ and get a new function $\Phi \in C^{\infty}(X)$. It is obvious that there exists a positive constant $\widetilde{C}$ such that

$$
(\sqrt{-1} \partial \bar{\partial} \Phi)(u, \bar{u}) \geq-\widetilde{C}|u|_{\omega}^{2}
$$

over $X$. Now we define $f=\frac{C}{2 \widetilde{C}} \Phi$; then $f$ satisfies (3.7) and (3.8) with $C_{1}=\frac{C \widetilde{C}_{1}}{2 \widetilde{C}}$.

We define a new smooth Hermitian metric $\widetilde{h}=e^{-f} h$ on $\mathscr{E}$. By formula (3.5), the curvature tensor $\widetilde{R}$ of $(\mathscr{E}, \widetilde{h})$ satisfies

$$
\widetilde{R}(u, \bar{u}, v, \bar{v})=e^{-f}\left(R(u, \bar{u}, v, \bar{v})+(\partial \bar{\partial} f)(u, \bar{u}) \cdot|v|_{h}^{2}\right)
$$

We claim that $(\mathscr{E}, \widetilde{h})$ is uniformly RC-positive over $X$. Indeed, for a point $q \in S$, since $(\mathscr{E}, h)$ is uniformly $\mathrm{RC}$-semi-positive at $q$, there exists a unit vector $u \in T_{q} X$ such that $R(u, \bar{u}, v, \bar{v}) \geq 0$ for all $v \in \mathscr{E}_{q}$. By estimate (3.8), we have

$$
\begin{aligned}
\widetilde{R}(u, \bar{u}, v, \bar{v}) & =e^{-f}\left(R(u, \bar{u}, v, \bar{v})+(\partial \bar{\partial} f)(u, \bar{u}) \cdot|v|_{h}^{2}\right) \\
& \geq e^{-f}(\partial \bar{\partial} f)(u, \bar{u}) \cdot|v|_{h}^{2} \\
& \geq C_{1} e^{-f}|v|_{h}^{2},
\end{aligned}
$$

for every $v \in \mathscr{E}_{q}$. For a point $q \in X \backslash S$, since $(\mathscr{E}, h)$ is uniformly RC-positive over $X \backslash S$, by formula (3.6) and Proposition 2.9, there exists some unit vector $u \in T_{q} X$ such that

$$
R(u, \bar{u}, v, \bar{v}) \geq C|v|_{h}^{2}
$$

for every $v \in \mathscr{E}_{q}$. On the other hand, by estimate (3.7), we have

$$
\widetilde{R}(u, \bar{u}, v, \bar{v})=e^{-f}\left(R(u, \bar{u}, v, \bar{v})+(\partial \bar{\partial} f)(u, \bar{u}) \cdot|v|_{h}^{2}\right) \geq \frac{C}{2} e^{-f}|v|_{h}^{2} .
$$

Hence, we conclude $(\mathscr{E}, \widetilde{h})$ is uniformly RC-positive over $X$.

The proof of Theorem 1.6. By Theorem 3.5, $T_{X}$ has a uniformly RC-positive metric $\widetilde{h}=e^{-f} \cdot h$. By Proposition 2.12, we know $\Lambda^{2} T_{X}$ is uniformly RC-positive. By Corollary 2.11 and Corollary $2.5, X$ is 
a projective manifold. On the other hand, by Theorem 3.3, for any line bundle $\mathscr{L}$ over $X$, there exists a positive constant $C=C(\mathscr{L})$ such that

$$
H^{0}\left(X,\left(T_{X}^{*}\right)^{\otimes m} \otimes \mathscr{L}^{\otimes k}\right)=0
$$

for all $m, k \in \mathbb{N}^{+}$with $m \geq C k$. Therefore, by a celebrated theorem of Campana, Demailly, and Peternell [9, Theorem 1.1], $X$ is rationally connected. Indeed, we deduce from (3.12) that for each $1 \leq p \leq \operatorname{dim} X$, any invertible sheaf $\mathcal{F} \subset \Omega_{X}^{p}$ is not pseudo-effective. Otherwise, if there exists a pseudo-effective invertible sheaf $\mathcal{F} \subset \Omega_{X}^{p}$, then there exists a very ample line bundle $A$ such that $H^{0}\left(X, \mathcal{F}^{\otimes \ell} \otimes A\right) \neq 0$ for all $\ell \geq 0$, and so $H^{0}\left(X, \operatorname{Sym}^{\otimes \ell} \Omega_{X}^{p} \otimes A\right) \neq 0$. Since for some large $m$, $\operatorname{Sym}^{\otimes \ell} \Omega_{X}^{p} \subset\left(T_{X}^{*}\right)^{\otimes m}$, we get a contradiction to (3.12), in particular when $p=n, K_{X}$ is not pseudo-effective. Thanks to [6], $X$ is uniruled. Let $\pi: X \rightarrow Z$ be the associated MRC fibration of $X$. After possibly resolving the singularities of $\pi$ and $Z$, we may assume that $\pi$ is a proper morphism and $Z$ is smooth. By a result of Graber, Harris, and Starr [19, Corollary 1.4], it follows that the target $Z$ of the MRC fibration is either a point or a positive dimensional variety that is not uniruled. Suppose $X$ is not rationally connected; then $\operatorname{dim} Z \geq 1$. Hence $Z$ is not uniruled; by [6] again, $K_{Z}$ is pseudo-effective. Since $K_{Z}=\Omega_{Z}^{\operatorname{dim}} Z \subset \Omega_{X}^{\operatorname{dim}} Z$ is pseudo-effective, we get a contradiction. Hence $X$ is rationally connected.

If $S=\varnothing$, we obtain Theorem 1.3. By using rational connectedness, one has

Corollary 3.6. Let $X$ be a compact Kähler manifold. If $X$ admits a smooth Hermitian metric $\omega$ such that $\left(T_{X}, \omega\right)$ is uniformly $R C$-positive, then there is no non-constant holomorphic map from $X$ to a compact complex manifold $Y$ without rational curves.

There are many compact complex manifolds that contain no rational curves. For instance, this is the case of manifolds $Y$ such that

$\circ Y$ is Kobayashi hyperbolic.

- $Y$ has a nef cotangent bundle.

- $Y$ has a Hermitian metric with non-positive holomorphic sectional curvature.

$\circ Y$ is an Abelian variety.

\section{RC-positive metrics on rationally connected manifolds}

In this section, we will discuss a general theory for uniformly RC-positive vector bundles and prove Theorem 1.4. Let's recall that a line bundle $\mathscr{L}$ is uniformly RC-positive if and only if it has a smooth Hermitian metric $h$ such that its curvature $-\sqrt{-1} \partial \bar{\partial} \log h$ has at least one positive eigenvalue everywhere. In [48, Theorem 1.4], we obtained an equivalent characterization for uniformly RC-positive line bundles.

Theorem 4.1. Let $\mathscr{L}$ be a holomorphic line bundle over a compact complex manifold X. The following statements are equivalent:

1. $\mathscr{L}$ is uniformly $R C$-positive.

2. The dual line bundle $\mathscr{L}^{*}$ is not pseudo-effective.

Two key ingredients in the proof of Theorem 4.1 are a conformal (exponential) perturbation method and an integration criterion for pseudo-effectiveness over compact complex manifolds (e.g. [27]). We refer to $[47,48]$ and the references therein. As an application of Theorem 4.1, we obtain the following result.

Corollary 4.2. Let $X$ be a projective manifold. If $\mathscr{L}$ is uniformly $R C$-semi-positive, then the dual line bundle $\mathscr{L}^{*}$ is not big. 
Proof. Let $\mathscr{A}$ be an ample line bundle over $X$. We argue by contradiction. Suppose $\mathscr{L}^{*}$ is big; then there exists a large number $k \in \mathbb{Z}_{+}$such that $\mathscr{L}^{* k} \otimes \mathscr{A}^{*}$ is big, and so it is pseudo-effective. By Theorem 4.1, $\mathscr{L}^{k} \otimes \mathscr{A}$ is not uniformly RC-positive, which is absurd.

The following concepts are generalizations of uniform RC-positivity for line bundles.

Definition 4.3. Let $\mathscr{L}$ be a line bundle over a compact complex manifold $X$.

1. $\mathscr{L}$ is called $q$-positive if there exists a smooth Hermitian metric $h$ on $\mathscr{L}$ such that the Chern curvature $R^{(\mathscr{L}, h)}=-\sqrt{-1} \partial \bar{\partial} \log h$ has at least $(\operatorname{dim} X-q)$ positive eigenvalues at every point on $X$.

2. $\mathscr{L}$ is called $q$-ample if for any coherent sheaf $\mathscr{F}$ on $X$, there exists a positive integer $m_{0}=$ $m_{0}(X, \mathscr{L}, \mathscr{F})>0$ such that

$$
H^{i}\left(X, \mathscr{F} \otimes \mathscr{L}^{m}\right)=0 \quad \text { for } i>q, m \geq m_{0}
$$

Note that the nontrivial range is $0 \leq q \leq \operatorname{dim} X-1$. By the celebrated Cartan-Serre-Grothendieck criterion for ampleness and Kodaira embedding theorem, one has

Theorem 4.4 (Cartan-Serre-Grothendieck, Kodaira). . Let $\mathscr{L} \rightarrow X$ be a holomorphic line bundle over a projective manifold $X$. Then the following statements are equivalent:

1. $\mathscr{L}$ is 0 -ample.

2. $\mathscr{L}$ is 0 -positive.

In [2, Theorem 14], Andreotti and Grauert proved the following fundamental theorem (see also [18, Proposition 2.1]).

Theorem 4.5. A q-positive line bundle is q-ample.

In [18], Demailly, Peternell, and Schneider systematically investigated partial vanishing theorems and proposed the following converse to the Andreotti-Grauert theorem:

Problem 4.6. On a projective manifold, if a line bundle is q-ample, is it q-positive?

This is a long-standing open problem. The key difficulty arises from constructing a precise metric according to the formal partial vanishing theorem (4.1). Recently, there has been some progress on this problem (see [14, 44, 31, 40, 4, 26, 20]). In complex dimension two, Demailly proved in [14] an asymptotic version of this converse to the Andreotti-Grauert theorem using tools related to the holomorphic Morse inequality; and S. Matsumura obtained in [31, Theorem 1.3] a positive answer to the question for projective surfaces. However, there exist higher-dimensional counterexamples to the converse Andreotti-Grauert problem for $q$ in the range $\frac{\operatorname{dim} X}{2}-1<q<\operatorname{dim} X-2$, constructed by Ottem [40, Theorem 10.3].

As a weak dual to the Cartan-Serre-Grothendieck-Kodaira theorem 4.4, we established in [48] that

Theorem 4.7. Let $\mathscr{L} \rightarrow X$ be a holomorphic line bundle over a projective manifold $X$. Then the following statements are equivalent:

1. $\mathscr{L}$ is $(\operatorname{dim} X-1)$-ample.

2. $\mathscr{L}$ is $(\operatorname{dim} X-1)$-positive.

3. $\mathscr{L}$ is uniformly $R C$-positive.

Let $\mathscr{E}$ be a vector bundle over $X$ and $\mathbb{P}\left(\mathscr{E}^{*}\right)$ be the projective bundle of $\mathscr{E}$. The points of the projective bundle $\mathbb{P}\left(\mathscr{E}^{*}\right)$ of $\mathscr{E} \rightarrow X$ can be identified with the hyperplanes of $\mathscr{E}$. Note that every hyperplane $V$ in $\mathscr{E}_{z}$ corresponds bijectively to the line of linear forms in $\mathscr{E}_{z}^{*}$ that vanish on $V$. Let $\pi: \mathbb{P}\left(\mathscr{E}^{*}\right) \rightarrow X$ be the natural projection. There is a tautological hyperplane sub-bundle $S$ of $\pi^{*} \mathscr{E}$ over $\mathbb{P}\left(\mathscr{E}^{*}\right)$ such that

$$
S_{[\xi]}=\xi^{-1}(0) \subset \mathscr{E}_{z}
$$


for all $\xi \in \mathscr{E}_{Z}^{*} \backslash\{0\}$. The quotient line bundle $\pi^{*} \mathscr{E} / S$ is denoted $\mathcal{O}_{\mathscr{C}}(1)$ and is called the tautological line bundle associated with $\mathscr{E} \rightarrow X$. Hence there is an exact sequence of vector bundles over $\mathbb{P}\left(\mathscr{E}^{*}\right)$

$$
0 \rightarrow S \rightarrow \pi^{*} \mathscr{E} \rightarrow \mathcal{O}_{\mathscr{E}}(1) \rightarrow 0
$$

A holomorphic vector bundle $\mathscr{E} \rightarrow X$ is called ample if the line bundle $\mathcal{O}_{\mathscr{E}}(1)$ is ample over $\mathbb{P}\left(\mathscr{E}^{*}\right)$.

Proposition 4.8. Let $(\mathscr{E}, h)$ be an RC-positive vector bundle over a compact complex manifold $X$. Then

1. $\mathcal{O}_{\mathscr{E}}(1)$ is $(\operatorname{dim} X-1)$-positive over $\mathbb{P}\left(\mathscr{E}^{*}\right)$.

2. $\mathcal{O}_{\mathscr{E} *}(1)$ is not pseudo-effective over $\mathbb{P}(\mathscr{E})$.

3. $\mathcal{O}_{\mathscr{C}} *(-1)$ is uniformly $R C$-positive over $\mathbb{P}(\mathscr{E})$.

Proof. It follows from the curvature formulas of $\mathcal{O}_{\mathscr{E} *}(-1)$ and $\mathcal{O}_{\mathscr{E}}(1)$ induced by $(\mathscr{E}, h)$ (e.g. [49, Proposition 4.1], [50, Proposition 2.6]) and Theorem 4.1. Indeed, for any Hermitian metric $h$ on $\mathscr{E}$, the induced Hermitian metric $g$ on the line bundle $\mathcal{O}_{\mathscr{E}}(1)$ has curvature form over $\mathbb{P}\left(\mathscr{E}^{*}\right)$

$$
R^{\mathcal{O}_{\mathscr{\delta}}(1)}=\sqrt{-1}\left(\sum R_{i \bar{j} \alpha \bar{\beta}}^{\mathscr{E}} \frac{a_{\beta} \bar{a}_{\alpha}}{|a|^{2}} d z^{i} \wedge d \bar{z}^{j}+\frac{1}{|a|^{2}} \sum_{A, B=1}^{r-1}\left(1-\frac{a_{B} \bar{a}_{A}}{|a|^{2}}\right) d w^{A} \wedge d \bar{w}^{B}\right)
$$

where $|a|^{2}=\sum_{\alpha=1}^{r}\left|a_{\alpha}\right|^{2}, a_{r}=1$, and $r=\operatorname{rank}(\mathscr{E})$. Hence, if $(\mathscr{E}, h)$ is RC-positive, then $\left(\mathcal{O}_{\mathscr{E}}(1), g\right)$ has one extra positive eigenvalue from the curvature term

$$
\sum R_{i \bar{j} \alpha \bar{\beta}}^{\mathscr{E}} \frac{a_{\beta} \bar{a}_{\alpha}}{|a|^{2}} d z^{i} \wedge d \bar{z}^{j}
$$

and $\left(\mathcal{O}_{\mathscr{E}}(1), g\right)$ is $(\operatorname{dim} X-1)$-positive, i.e. $\left(\mathcal{O}_{\mathscr{E}}(1), g\right)$ has at least $r$ positive eigenvalues everywhere, i.e. $\mathcal{O}_{\mathscr{E}}(1)$ is $(\operatorname{dim} X-1)$-positive. Similarly, $\mathcal{O}_{\mathscr{C}}(-1)$ has curvature form over $\mathbb{P}(\mathscr{E})$

$$
R^{\mathcal{O}_{\mathscr{G} *}(-1)}=\sqrt{-1}\left(\sum R_{i \bar{j} \alpha \bar{\beta}}^{\mathscr{E}} \frac{a_{\beta} \bar{a}_{\alpha}}{|a|^{2}} d z^{i} \wedge d \bar{z}^{j}-\frac{1}{|a|^{2}} \sum_{A, B=1}^{r-1}\left(1-\frac{a_{B} \bar{a}_{A}}{|a|^{2}}\right) d w^{A} \wedge d \bar{w}^{B}\right) .
$$

We deduce that if $(\mathscr{E}, h)$ is RC-positive, then $\mathcal{O}_{\mathscr{E}}(-1)$ is uniformly RC-positive.

By adapting ideas in [48], we can show that three conclusions in Proposition 4.8 are equivalent on projective manifolds.

Theorem 4.9. Let $\mathscr{E}$ be a holomorphic vector bundle over a projective manifold X. Then the following statements are equivalent:

1. $\mathcal{O}_{\mathscr{E}}(1)$ is $(\operatorname{dim} X-1)$-ample.

2. $\mathcal{O}_{\mathscr{C} *}(1)$ is not pseudo-effective.

3. $\mathcal{O}_{\mathscr{C}^{*}}(-1)$ is uniformly $R C$-positive.

Proof. (1) $\Longrightarrow(2)$. If $\mathcal{O}_{\mathscr{C}}(1)$ is $(\operatorname{dim} X-1)$-ample, we deduce that $\mathcal{O}_{\mathscr{C}}(1)$ is not pseudo-effective. Otherwise, if $\mathcal{O}_{\mathscr{E}} *(1)$ is pseudo-effective, it is well known that there exists an ample line bundle $\mathscr{L}$ on $\mathbb{P}(\mathscr{E})$ such that

$$
H^{0}\left(\mathbb{P}(\mathscr{E}), \mathcal{O}_{\mathscr{C}}(m) \otimes \mathscr{L}\right) \neq 0 \quad \text { for all } m \geq 0
$$

More precisely, $\mathscr{L}$ can be chosen in such a way: fix a very ample line bundle $\mathscr{H}$ over $\mathbb{P}(\mathscr{E})$, and if $\mathscr{L}$ is an ample line bundle such that

$$
\mathscr{L} \otimes K_{\mathbb{P}(\mathscr{E})}^{-1} \otimes \mathscr{H}^{-(\operatorname{dim} \mathbb{P}(\mathscr{E})+1)}
$$


is ample, then (4.4) holds. We can choose $\mathscr{L}$ in a special form

$$
\mathscr{L}=\mathcal{O}_{\mathscr{C}} *\left(m_{0}\right) \otimes \pi^{*}\left(\mathscr{A}^{\otimes m_{1}}\right)
$$

where $\mathscr{A}$ is an ample line bundle over $X$, and $m_{0}, m_{1}$ are two large positive integers with $m_{1} \gg m_{0}$, and $\pi: \mathbb{P}(\mathscr{E}) \rightarrow X$ is the projection. For the reader's convenience, we write down the construction explicitly. Indeed, for large $k, \mathscr{E}^{*} \otimes \mathscr{A}^{\otimes k}$ is an ample vector bundle over $X$, i.e. $\mathcal{O}_{\mathscr{E}}(1) \otimes \pi^{*}\left(\mathscr{A}^{\otimes k}\right)$ is an ample line bundle over $\mathbb{P}(\mathscr{E})$. There exists a large $k_{1}$ such that $\mathscr{H}=\mathcal{O}_{\mathscr{C}^{*}}\left(k_{1}\right) \otimes \pi^{*}\left(\mathscr{A}^{\otimes\left(k k_{1}\right)}\right)$ is a very ample line bundle over $\mathbb{P}(\mathscr{E})$. On the other hand,

$$
K_{\mathbb{P}(\mathscr{E})}=\mathcal{O}_{\mathscr{C}}(-r) \otimes \pi^{*}\left(K_{X}\right) \otimes \pi^{*}\left(\operatorname{det} \mathscr{E}^{*}\right)
$$

where $r$ is the rank of $\mathscr{E}$. There exists a positive number $k_{2}$ such that

$$
\mathscr{A}^{\otimes k_{2}} \otimes K_{X}^{-1} \otimes \operatorname{det} \mathscr{E}
$$

is ample over $X$. Now we can take

$$
m_{0}=k_{1}(n+r)-r+1 \quad \text { and } \quad m_{1}=k_{2}+k k_{1}(n+r)+k
$$

and so

$$
\mathscr{L} \otimes K_{\mathbb{P}(\mathscr{C})}^{-1} \otimes \mathscr{H}^{-(n+r)}=\mathcal{O}_{\mathscr{C} *}(1) \otimes \pi^{*}\left(\mathscr{A}^{\otimes k}\right) \otimes \pi^{*}\left(\mathscr{A}^{\otimes k_{2}} \otimes K_{X}^{-1} \otimes \operatorname{det} \mathscr{E}\right)
$$

is ample over $\mathbb{P}(\mathscr{E})$.

Therefore, by the Le Potier isomorphism, (4.4) is equivalent to

$$
H^{0}\left(X, \operatorname{Sym}^{\otimes k} \mathscr{E}^{*} \otimes \mathscr{A}^{\otimes m_{1}}\right) \neq 0
$$

for large $k$. Applying the Serre duality on $X$ and the Le Potier isomorphism again, we obtain

$$
H^{n}\left(\mathbb{P}\left(\mathscr{E}^{*}\right), \mathcal{O}_{\mathscr{E}}(k) \otimes \pi_{1}^{*}\left(\left(\mathscr{A}^{*}\right)^{\otimes m_{1}}\right) \otimes \Omega_{\mathbb{P}\left(\mathscr{E}^{*}\right)}^{n}\right) \neq 0
$$

for large $k$, where $\pi_{1}: \mathbb{P}\left(\mathscr{E}^{*}\right) \rightarrow X$ is the projection. Let $\mathscr{F}=\pi_{1}^{*}\left(\left(\mathscr{A}^{*}\right)^{\otimes m_{1}}\right) \otimes \Omega_{\mathbb{P}(\mathscr{C} *)}^{n}$, and we know $\mathcal{O}_{\mathscr{E}}(1)$ cannot be $(n-1)$-ample. This is a contradiction. Hence $\mathcal{O}_{\mathscr{E} *}(1)$ is not pseudo-effective.

The equivalence of (2) and (3) follows from Theorem 4.1.

(3) $\Longrightarrow(1)$. We need to show that for any line bundle $\mathscr{F}$ over $\mathbb{P}\left(\mathscr{E}^{*}\right)$, there exists $k_{0}>0$ such that when $k>k_{0}$, one has

$$
H^{n}\left(\mathbb{P}\left(\mathscr{E}^{*}\right), \mathcal{O}_{\mathscr{E}}(k) \otimes \mathscr{F}\right)=0
$$

It is well known that $\mathscr{F}$ takes the form $\mathcal{O}_{\mathscr{C}}(m) \otimes \pi_{1}^{*}(\mathscr{L})$, where $m \in \mathbb{Z}$, and $\mathscr{L}$ is a line bundle over $X$, and $\pi: \mathbb{P}\left(\mathscr{E}^{*}\right) \rightarrow X$ is the projection. Since $\mathcal{O}_{\mathscr{E}^{*}}(-1)$ is uniformly RC-positive, by Theorem 3.3 , there exists $k_{1}>0$ such that

$$
\mathcal{O}_{\mathscr{C} *}(-k) \otimes \pi^{*}\left(\mathscr{L} \otimes K_{X}^{-1}\right)
$$

is also uniformly RC-positive when $k \geq k_{1}$, where $\pi: \mathbb{P}(\mathscr{E}) \rightarrow X$ is the projection. By Theorem 2.4,

$$
H^{0}\left(\mathbb{P}(\mathscr{E}), \mathcal{O}_{\mathscr{E}}(k) \otimes \pi^{*}\left(\mathscr{L}^{*} \otimes K_{X}\right)\right)=0
$$

for $k>k_{1}$. By Le Potier isomorphism and Serre duality, we deduce

$$
H^{0}\left(X, \operatorname{Sym}^{\otimes k} \mathscr{E}^{*} \otimes \mathscr{L}^{*} \otimes K_{X}\right) \cong H^{n}\left(X, \operatorname{Sym}^{\otimes k} \mathscr{E} \otimes \mathscr{L}\right)=0 .
$$


Hence, we have

$$
H^{n}\left(\mathbb{P}\left(\mathscr{E}^{*}\right), \mathcal{O}_{\mathscr{E}}(k) \otimes \pi_{1}^{*} \mathscr{L}\right)=0
$$

for $k>k_{1}$. The proof of (4.7) is completed.

On the other hand, we have

Proposition 4.10. Let $X$ be a projective manifold. Suppose $\mathcal{O}_{\mathscr{E}}(1)$ is $(\operatorname{dim} X-1)$-ample; then the restriction of $\mathcal{O}_{\mathscr{E}}(1)$ to every smooth sub-manifold $Y \subset \mathbb{P}\left(\mathscr{E}^{*}\right)$ with $\operatorname{dim} Y=\operatorname{dim} X$ is $(\operatorname{dim} X-1)$ positive.

Proof. Let $f: Y \rightarrow \mathbb{P}\left(\mathscr{E}^{*}\right)$ be the inclusion map. Using the projection formula and the Leray spectral sequence, one has

$$
H^{i}\left(Y, \mathscr{F} \otimes\left(f^{*} \mathcal{O}_{\mathscr{E}}(m)\right)\right)=H^{i}\left(\mathbb{P}\left(\mathscr{E}^{*}\right), f_{*}(\mathscr{F}) \otimes \mathcal{O}_{\mathscr{E}}(m)\right)
$$

Hence, if $\mathcal{O}_{\mathscr{E}}(1) \rightarrow \mathbb{P}\left(\mathscr{E}^{*}\right)$ is $(\operatorname{dim} X-1)$-ample, $f^{*}\left(\mathcal{O}_{\mathscr{C}}(1)\right) \rightarrow Y$ is also $(\operatorname{dim} X-1)$-ample. On the other hand, since $\operatorname{dim} Y=\operatorname{dim} X$, by Theorem 4.7, the $(\operatorname{dim} X-1)$-ample line bundle $f^{*}\left(\mathcal{O}_{\mathscr{E}}(1)\right)$ over $Y$ is $(\operatorname{dim} X-1)$-positive.

Motivated by these properties, we propose the following conjecture.

Conjecture 4.11. Let $\mathscr{E}$ be a holomorphic vector bundle over a projective manifold X. Then the following statements are equivalent:

1. $\mathcal{O}_{\mathscr{C}} *(-1)$ is $R C$-positive.

2. $\mathcal{O}_{\mathscr{E}}(1)$ is $(\operatorname{dim} X-1)$-ample.

3. $\mathcal{O}_{\mathscr{E}}(1)$ is $(\operatorname{dim} X-1)$-positive.

4. $\mathscr{E}$ is RC-positive.

Note that the implications (4) $\Longrightarrow(3) \Longrightarrow(2) \Longleftrightarrow(1)$ in Conjecture 4.11 are known by Proposition 4.8, [2] and Theorem 4.9. On the other hand, when $\operatorname{rank}(\mathscr{E})=1$ or $\operatorname{dim} X=1$, Conjecture 4.11 is true by Theorem 4.7 and [10]. Note also that Conjecture 4.11 is analogous to a conjecture of P. Griffiths:

Conjecture 4.12. Let $\mathscr{E}$ be a vector bundle over a projective manifold X. Then the following statements are equivalent:

1. $\mathcal{O}_{\mathscr{E}}(1)$ is positive.

2. $\mathscr{E}$ is Griffiths positive.

It is easy to see that if $\mathscr{E}$ is Griffiths positive, then so is $\mathcal{O}_{\mathscr{E}}(1)$.

As an application of Theorem 4.9, we obtain a differential geometric characterization of rationally connected manifolds.

Theorem 4.13. Let $X$ be a projective manifold. Then the following statements are equivalent:

1. $X$ is rationally connected.

2. The line bundle $\mathcal{O}_{\Lambda^{p}} T_{X}^{*}(-1)$ is uniformly RC-positive for every $1 \leq p \leq \operatorname{dim} X$.

Proof. If $X$ is rationally connected, then by a variant of [9, Theorem 1.1] (e.g. [8, Proposition 1.4]), for any ample line bundle $\mathscr{L}$ on $X$, there exists a positive integer $m_{0}$ such that

$$
H^{0}\left(X, \operatorname{Sym}^{\otimes m}\left(\Lambda^{p} T_{X}^{*}\right) \otimes \mathscr{L}^{\otimes k}\right)=0
$$

for $m \geq m_{0} k$ and all $1 \leq p \leq \operatorname{dim} X$. We claim $\mathcal{O}_{\Lambda^{p}} T_{X}^{*}(-1)$ is uniformly RC-positive. Otherwise, by Theorem 4.9, $\mathcal{O}_{\Lambda^{p}} T_{X}^{*}(1)$ is pseudo-effective. Hence, by using a similar proof as in Theorem 4.9, we can 
find an ample line bundle $\mathscr{A}$ over $X$ such that (4.6) holds for $\mathscr{E}=\Lambda^{p} T_{X}$ : that is,

$$
H^{0}\left(X, \operatorname{Sym}^{\otimes m}\left(\Lambda^{p} T_{X}^{*}\right) \otimes \mathscr{A}^{\otimes m_{1}}\right) \neq 0
$$

for all large $m$. This is a contradiction.

On the other hand, if $\mathcal{O}_{\Lambda^{p} T_{X}^{*}}(-1)$ is uniformly RC-positive over $\mathbb{P}\left(\Lambda^{p} T_{X}\right)$, then by the Le Potier isomorphism and Theorem 3.3, for any coherent sheaf of the form $\mathscr{F}=\pi^{*}\left(\mathscr{L}^{\otimes k}\right)$ over $\mathbb{P}\left(\Lambda^{p} T_{X}\right)$, we have

$$
H^{0}\left(X, \operatorname{Sym}^{\otimes m}\left(\Lambda^{p} T_{X}^{*}\right) \otimes \mathscr{L}^{\otimes k}\right) \cong H^{0}\left(\mathbb{P}\left(\Lambda^{p} T_{X}\right), \mathcal{O}_{\Lambda^{p}} T_{X}^{*}(m) \otimes \mathscr{F}\right)=0
$$

for large $m$. Therefore, by the proof of [49, Theorem 1.4] (see also [9, Theorem 1.1] or [8, Proposition $1.4]), X$ is rationally connected.

As motivated by Theorem 4.9, Theorem 4.13, and Conjecture 4.11, we propose the following:

Problem 4.14. Let $X$ be a projective manifold. Suppose $X$ is rationally connected. Does $T_{X}$ admit an $R C$-positive Hermitian metric?

More generally, we expect the following.

Problem 4.15. The following statements are equivalent on a projective manifold $X$ :

1. $X$ is rationally connected.

2. $T_{X}$ is $R C$-positive.

3. $T_{X}$ is uniformly $R C$-positive.

In [49, Corollary 1.5], we obtain the following result.

Corollary 4.16. Let $X$ be a compact Kähler manifold. If there exist a Hermitian metric $\omega$ on $X$ and $a$ (possibly different) Hermitian metric $h$ on $T_{X}$ such that

$$
\operatorname{tr}_{\omega} R^{\left(T_{X}, h\right)} \in \Gamma\left(X, \operatorname{End}\left(T_{X}\right)\right)
$$

is positive definite, then $X$ is projective and rationally connected.

In particular, by the celebrated Calabi-Yau theorem [53], one gets the classical result of Campana [7] and Kollár-Miyaoka-Mori [25] that Fano manifolds are rationally connected. We propose a conjecture converse to Corollary 4.16, which is also analogous to the classical fact: a compact complex manifold is Fano if and only if it has a Hermitian metric with positive first Chern-Ricci curvature.

Problem 4.17. The following statements are equivalent on a projective manifold $X$ :

1. $X$ is rationally connected.

2. There exist a Hermitian metric $\omega$ on $X$ and a (possibly different) Hermitian metric $h$ on $T_{X}$ such that $\operatorname{tr}_{\omega} R^{\left(T_{X}, h\right)}$ is positive definite.

Remark 4.18. Problem 4.17 is also known to J.-P. Demailly [15]. A positive solution to Problem 4.17 gives an affirmative answer to Problem 4.14.

\section{Compact Kähler manifolds with non-negative holomorphic sectional curvature}

A compact Kähler manifold $(X, \omega)$ has positive (respectively, non-negative) holomorphic sectional curvature if for any nonzero vector $\xi=\left(\xi^{1}, \cdots, \xi^{n}\right)$,

$$
\left.R_{i \bar{j} k} \xi^{i} \bar{\xi}^{j} \xi^{k} \bar{\xi}^{\ell}>0 \quad \text { (respectively, } \geq 0\right)
$$


at each point of $X$. The negativity and non-positivity of the holomorphic sectional curvature can be defined in a similar way.

Theorem 5.1. Let $(X, \omega)$ be a compact Kähler manifold with positive (respectively, non-negative) holomorphic sectional curvature; then $\left(T_{X}, \omega\right)$ is uniformly $R C$-positive (respectively, uniformly $R C$ semi-positive).

Proof. Let $\kappa$ be the positive holomorphic sectional curvature of $(X, \omega)$, i.e.

$$
\kappa=\inf _{q \in X} \inf _{U \in T_{q} X \backslash\{0\}} \frac{R(U, \bar{U}, U, \bar{U})}{|U|^{4}} .
$$

For any point $q \in X$, let $e \in T_{q} X$ be a unit vector such that

$$
R(e, \bar{e}, e, \bar{e})=\inf _{U \in T_{q} X \backslash\{0\}} \frac{R(U, \bar{U}, U, \bar{U})}{|U|^{4}} .
$$

We know $R(e, \bar{e}, e, \bar{e}) \geq \kappa>0$. On the other hand, by [49, Lemma 6.1], for any unit vector $W \in T_{q} X$, we have

$$
2 R(e, \bar{e}, W, \bar{W}) \geq\left(1+|\langle W, e\rangle|^{2}\right) R(e, \bar{e}, e, \bar{e}) \geq \kappa .
$$

Hence, for any vector $v \in T_{q} X$, we obtain

$$
R(e, \bar{e}, v, \bar{v}) \geq \frac{\kappa}{2}|v|^{2},
$$

and so $\left(T_{X}, \omega\right)$ is uniformly RC-positive.

Remark 5.2. If a compact Kähler manifold $(X, \omega)$ has negative (respectively, non-positive) holomorphic sectional curvature, then $\left(T_{X}, \omega\right)$ is uniformly RC-negative (respectively, uniformly RC-non-positive).

As an application of Theorem 5.1 and Theorem 1.6, we obtain Theorem 1.7, i.e.

Theorem 5.3. Let $(X, \omega)$ be a compact Kähler manifold with non-negative holomorphic sectional curvature. If $S$ is open subset of $X$ such that $\left(T_{X}, \omega\right)$ is uniformly $R C$-positive over $X \backslash S$, and $\bar{S}$ is contained in some strongly pseudoconvex domain of $X$, then $X$ has a uniformly $R C$-positive Hermitian metric. Moreover, $X$ is a projective and rationally connected manifold.

Corollary 5.4. Let $(X, \omega)$ be a compact Kähler manifold with positive holomorphic sectional curvature. Then $\left(T_{X}, \omega\right)$ is uniformly $R C$-positive. Moreover, $X$ is projective and rationally connected. In particular, $X$ is simply connected.

Remark 5.5. It is not hard to see that Theorem 5.3 can also hold under certain weaker conditions. For related topics on holomorphic sectional curvature, we refer to [21, 1, 46, 29, 3, 45, 13, 32] and the references therein.

\section{RC-positive Finsler vector bundles over complex manifolds}

Let $\mathscr{E}$ be a holomorphic vector bundle over a complex manifold $X$ with complex $\operatorname{rank} r$. Let $\left(z^{1}, \cdots, z^{n}\right)$ be the local holomorphic coordinates on $X$ and $\left(w^{1}, \cdots, w^{r}\right)$ be the holomorphic coordinates on the fiber of $\mathscr{E}$. Let $\mathscr{E} \backslash\{0\}$ be the complement of the zero section of $\mathscr{E}$.

Definition 6.1. A pseudoconvex complex Finsler metric $\mathfrak{F}$ on $\mathscr{E}$ is a continuous function $\mathfrak{F}: \mathscr{E} \rightarrow$ $[0,+\infty)$ satisfying the following:

1. $\mathfrak{F}$ is smooth on $\mathscr{E} \backslash\{0\}$.

2. $\mathfrak{F}(z, w)>0$ for all $w \neq 0$. 
3. $\mathfrak{F}(z, \lambda w)=|\lambda|^{2} \mathfrak{F}(z, w)$ for all $\lambda \in \mathbb{C}$.

4. The $(r \times r)$ Hermitian matrix $\left(\frac{\partial^{2} \mathscr{F}}{\partial w^{\alpha} \partial \bar{w}^{\beta}}\right)$ is positive definite over $\mathscr{E} \backslash\{0\}$.

Let $\mathfrak{F}$ be a pseudoconvex complex Finsler metric on $\mathscr{E} \rightarrow X$. It is well known that

$$
\left(h_{\alpha \bar{\beta}}\right)=\left(\frac{\partial^{2} \mathfrak{F}}{\partial w^{\alpha} \partial \bar{w}^{\beta}}\right)
$$

defines a smooth Hermitian metric on the holomorphic vector bundle $\pi^{*} \mathscr{E} \rightarrow \mathbb{P}\left(\mathscr{E}^{*}\right)$, where $\pi$ : $\mathbb{P}\left(\mathscr{E}^{*}\right) \rightarrow X$ is the projection. Note that, in general, $\mathfrak{F}$ does not give a Hermitian metric on $\mathscr{E} \rightarrow X$.

Definition 6.2. Let $\mathfrak{F}$ be a complex Finsler metric on $\mathscr{E} \rightarrow X$. $(\mathscr{E}, \mathfrak{F})$ is called an RC-positive (respectively, a uniformly RC-positive) Finsler vector bundle if $\mathfrak{F}$ is pseudoconvex and the induced Hermitian vector bundle $\left(\pi^{*} \mathscr{E}, h\right)$ is RC-positive (respectively, uniformly RC-positive) over $\mathbb{P}\left(\mathscr{E}^{*}\right)$.

Remark 6.3. If $(\mathscr{E}, h)$ is a Hermitian holomorphic vector bundle, then it induces a pseudoconvex Finsler metric $\mathfrak{F}$ on $\mathscr{E}$. Moreover, if $(\mathscr{E}, h)$ is RC-positive (respectively, uniformly RC-positive), then ( $\mathscr{E}, \mathfrak{F})$ is RC-positive (respectively, uniformly RC-positive).

Theorem 6.4. Let $X$ be a compact complex manifold. Suppose $(\mathscr{E}, \mathfrak{F})$ is an RC-positive Finsler vector bundle. Then we have

$$
H^{0}\left(X, \operatorname{Sym}^{\otimes m_{\mathscr{E}}}\right)=0, \text { for all } \quad m \geq 1 .
$$

Moreover, for any line bundle $\mathscr{L} \rightarrow X$, there exists a positive constant $c \mathscr{L}$ such that

$$
H^{0}\left(X, \operatorname{Sym}^{\otimes m} \mathscr{C}^{*} \otimes \mathscr{L}^{* \otimes k}\right)=0
$$

for all positive integers $m, k$ with $m \geq c_{\mathscr{L} k}$.

Proof. If $(\mathscr{E}, \mathfrak{F})$ is an RC-positive Finsler bundle, then the induced Hermitian vector bundle $\left(\pi^{*} \mathscr{E}, h\right)$ is RC-positive, where $\pi: \mathbb{P}\left(\mathscr{E}^{*}\right) \rightarrow X$ is the projection. Let $\mathscr{F}=\pi^{*}(\mathscr{E})$ and $Y=\mathbb{P}\left(\mathscr{E}^{*}\right)$. By Proposition 4.8, $\mathcal{O}_{\mathscr{F} *}(-1)$ is an RC-positive line bundle over the projective bundle $\widetilde{\pi}: \mathbb{P}(\mathscr{F}) \rightarrow Y$. Hence, by Theorem 3.3, we have

$$
H^{0}\left(\mathbb{P}(\mathscr{F}), \mathcal{O}_{\mathscr{F} *}(m) \otimes \widetilde{\pi}^{*}\left(\pi^{*}\left(\mathscr{L}^{* \otimes k}\right)\right)\right)=0
$$

for all positive integers $m, k$ with $m \geq c_{\mathscr{L} k}$. By the Le Potier isomorphism, we have

$$
\begin{aligned}
H^{0}\left(X, \operatorname{Sym}^{\otimes m} \mathscr{E}^{*} \otimes \mathscr{L}^{\otimes k}\right) & \cong H^{0}\left(\mathbb{P}\left(\mathscr{C}^{*}\right), \pi^{*}\left(\operatorname{Sym}^{\otimes m} \mathscr{E}^{*}\right) \otimes \pi^{*}\left(\mathscr{L}^{\otimes k}\right)\right) \\
& \cong H^{0}\left(\mathbb{P}\left(\mathscr{E}^{*}\right), \operatorname{Sym}^{\otimes m} \pi^{*}\left(\mathscr{E}^{*}\right) \otimes \pi^{*}\left(\mathscr{L}^{\otimes k}\right)\right) \\
& =H^{0}\left(Y, \operatorname{Sym}^{\otimes m} \mathscr{F}^{*} \otimes \pi^{*}\left(\mathscr{L}^{* \otimes k}\right)\right) \\
& \cong H^{0}\left(\mathbb{P}(\mathscr{F}), \mathcal{O}_{\mathscr{F}^{*}}(m) \otimes \tilde{\pi}^{*}\left(\pi^{*}\left(\mathscr{L}^{* \otimes k}\right)\right)\right) \\
& =0 .
\end{aligned}
$$

If we take $\mathscr{L}$ to be a trivial line bundle, then there exists a large positive integer $m$ such that $H^{0}\left(X, \operatorname{Sym}^{\otimes m} \mathscr{C}^{*}\right)=0$. It is easy to see that $H^{0}\left(X, \mathscr{E}^{*}\right)=0$ and so $H^{0}\left(X, \operatorname{Sym}^{\otimes m} \mathscr{E}^{*}\right)=0$ for all $m \geq 1$.

Theorem 6.5. Let $X$ be a compact complex manifold. Suppose $(\mathscr{E}, \mathfrak{F})$ is a uniformly RC-positive Finsler vector bundle. Then we have

$$
H^{0}\left(X,\left(\mathscr{E}^{*}\right)^{\otimes m}\right)=0, \text { for all } m \geq 1 .
$$


Moreover, for any line bundle $\mathscr{L} \rightarrow X$, there exists a positive constant $c_{\mathscr{L}}$ such that

$$
H^{0}\left(X,\left(\mathscr{E}^{*}\right)^{\otimes m} \otimes \mathscr{L}^{\otimes k}\right)=0
$$

for all positive integers $m, k$ with $m \geq c_{\mathscr{L} k}$.

Proof. The induced Hermitian vector bundle $\left(\pi^{*} \mathscr{E}, h\right)$ is uniformly RC-positive. By Proposition 2.12, $\pi^{*}\left(\mathscr{E}^{\otimes m}\right) \cong\left(\pi^{*}(\mathscr{E})\right)^{\otimes m}$ is uniformly RC-positive for all $m \geq 1$. On the other hand,

$$
H^{0}\left(X,\left(\mathscr{C}^{*}\right)^{\otimes m} \otimes \mathscr{L}^{\otimes k}\right) \cong H^{0}\left(\mathbb{P}\left(\mathscr{C}^{*}\right),\left(\pi^{*}\left(\mathscr{C}^{*}\right)\right)^{\otimes m} \otimes \pi^{*}\left(\mathscr{L}^{\otimes k}\right)\right) .
$$

Hence, Theorem 6.5 follows from Theorem 3.3.

As an application of Theorem 6.4 and [49, Theorem 1.4], we obtain

Theorem 6.6. Let $X$ be a compact Kähler manifold of complex dimension $n$. Suppose that for every $1 \leq p \leq n$, there exists a Finsler metric $\mathfrak{F}_{p}$ on $\Lambda^{p} T_{X}$ such that $\left(\Lambda^{p} T_{X}, \mathfrak{F}_{p}\right)$ is RC-positive; then $X$ is projective and rationally connected.

Similarly, as an application of Theorem 6.5, we obtain the following generalization of Theorem 1.3.

Theorem 6.7. Let $X$ be a compact Kähler manifold. If $X$ admits a Finsler metric $\mathfrak{F}$ such that $\left(T_{X}, \mathfrak{F}\right)$ is uniformly $R C$-positive, then $X$ is projective and rationally connected.

Remark 6.8. It is well known that there exists a one-to-one correspondence between the set of Hermitian metrics on $\mathcal{O}_{\mathscr{E}}(-1)$ and the set of Finsler metrics on $\mathscr{E}^{*}$ or $\mathscr{E}$. We can also define that a Finsler vector bundle $(\mathscr{E}, \mathfrak{F})$ is RC-positive if the induced Hermitian metric on $\mathcal{O}_{\mathscr{E}}(-1)$ is RC-positive. In this case, similar results as in Theorem 6.6 and Theorem 6.7 can be obtained.

Acknowledgements. I am very grateful to Professor K.-F. Liu and Professor S.-T. Yau for their support, encouragement, and stimulating discussions over the years. I would also like to thank Professors J.-Y. Cao, J.-P. Demailly, H.-T. Feng, Y.-H. Wu, J. Xiao, and X.-Y. Zhou for helpful suggestions.

\section{References}

[1] A. Alvarez, A. Chaturvedi, and G. Heier, 'Optimal pinching for the holomorphic sectional curvature of Hitchin's metrics on Hirzebruch surfaces', Contemp. Math., 133-142 (2015).

[2] A. Andreotti and H. Grauert, Théorème de finitude pour la cohomologie des espaces complexes . Bull. Soc. Math. France 90 (1962), 193-259.

[3] A. Alvarez, G. Heier, and F.-Y. Zheng, 'On projectivized vector bundles and positive holomorphic sectional curvature', Proc. Amer. Math. Soc. 146 (2018), 2877-2882.

[4] M.-V. Brown, 'Big $q$-ample line bundles', Compos. Math. 148(3) (2012), 790-798.

[5] Y. Brunebarbe and F. Campana, 'Fundamental group and pluri-differentials on compact Kähler manifolds', Mosc. Math. J. 16(4) (2016), 651-658.

[6] S. Boucksom, J.-P. Demailly, M. Paun, and P. Peternell, 'The pseudoeffective cone of a compact Kähler manifold and varieties of negative Kodaira dimension', J. Algebraic Geom. 22 (2013), 201-248.

[7] F. Campana, Connexité rationnelle des variétés de Fano, Ann. Sci. Ecole Norm. Sup. (4) 25 (1992), no. 5, $539-545$.

[8] F. Campana, 'Slope rational connectedness for orbifolds', arXiv:1607.07829.

[9] F. Campana,J.-P. Demailly, and T. Peternell, 'Rationally connected manifolds and semipositivity of the Ricci curvature', in Recent advances in Algebraic Geometry, LMS Lecture Notes Series 417, (2014), 71-91.

[10] F. Campana and H. Flenner, 'A characterization of ample vector bundles on a curve', Math. Ann. 287 (1990), no. 4, $571-575$.

[11] F. Campana and M. Paun, 'Positivity properties of the bundle of logarithmic tensors on compact Kähler manifolds', Compositio Math. 152 (2016) 2350-2370.

[12] J.-Y. Cao, 'Höring, A.. A decomposition theorem for projective manifolds with nef anticanonical bundle', J. Algebraic Geom. 28 (2019), no. 3, 567-597.

[13] A. Chaturvedi and G. Heier, 'Hermitian metrics of positive holomorphic sectional curvature on fibrations', Math. Z. 295(1-2) (2020), 349-364.

[14] J.-P. Demailly, 'A converse to the Andreotti-Grauert theorem', Ann. Fac. Sci. Toulouse Math . (6) 20 (2011), Fascicule Special, 123-135. 
[15] J.-P. Demailly, private communication.

[16] J.-P. Demailly, T. Peternell, and M. Schneider, 'Holomorphic line bundles with partially vanishing cohomology', in Proceedings of the Hirzebruch 65 Conference on Algebraic Geometry (Ramat Gan, 1993), 165-198, Israel Math. Conf. Proc., 9 (Bar-Ilan Univ., 1996).

[17] J.-P. Demailly, T. Peternell, and M. Schneider, 'Compact Kähler manifolds with hermitian semipositive anticanonical bundle,' Compositio Math. 101 (1996), 217-224.

[18] J.-P. Demailly, T. Peternell, and M. Schneider, 'Holomorphic line bundles with partially vanishing cohomology', in Proceedings of the Hirzebruch 65 Conference on Algebraic Geometry (Ramat Gan, 1993), 165-198, Israel Math. Conf. Proc., 9 (Bar-Ilan Univ., 1996).

[19] T. Graber, J. Harris, and J. Starr, 'Families of rationally connected varieties', J. Amer. Math. Soc. 16 (2003), no. 1, $57-67$.

[20] D. Greb and A. Küronya, 'Partial positivity: geometry and cohomology of $q$-ample line bundles', in Recent advances in algebraic geometry, 207-239, London Math. Soc. Lecture Note Ser. 417 (Cambridge Univ. Press, Cambridge, 2015).

[21] G. Heier and B. Wong, 'On projective Kähler manifolds of partially positive curvature and rational connectedness', arXiv: 1509.02149 .

[22] D. Huybrechts, Complex geometry. An introduction, Universitext (Springer-Verlag, Berlin, 2005).

[23] K. Kodaira, 'On Kähler varieties of restricted type (an intrinsic characterization of algebraic varieties)', Ann. of Math. (2) 60, (1954). 28-48.

[24] J. Kollár, Rational curves on algebraic varieties (Springer-Verlag, Berlin, 1996).

[25] J. Kollár, Y. Miyaoka, and S. Mori, 'Rationally connected varieties', J. Algebraic Geom. 1 (1992), no. 3, 429-448.

[26] A. Küronya, 'Positivity on subvarieties and vanishing of higher cohomology', Ann. Inst. Fourier 63 (2013) $1717-1737$.

[27] A. Lamari, 'Courants kähleriens et surfaces compactes', Ann. Inst. Fourier 49 (1999), 263-285.

[28] V. Lazic and T. Peternell, 'Rationally connected varieties on a conjecture of Mumford', Sci. China Math. 60 (2017), 10191028.

[29] G. Liu, 'Three-circle theorem and dimension estimate for holomorphic functions on Kähler manifolds', Duke Math. J. 165 (2016), no. 15, 2899-2919.

[30] K.-F. Liu and X.-K. Yang, 'Ricci curvatures on Hermitian manifolds', Trans. Amer. Math. Soc. 369 (2017), 5157-5196.

[31] S. Matsumura, 'Asymptotic cohomology vanishing and a converse to the Andreotti-Grauert theorem on surfaces', Ann. Inst. Fourier 63 (2013), 2199-2221.

[32] S. Matsumura, 'On the image of MRC fibrations of projective manifolds with semi-positive holomorphic sectional curvature', arXiv:1801.09081.

[33] S. Matsumura, 'On projective manifolds with semi-positive holomorphic sectional curvature', arXiv:1811.04182.

[34] N. Mok, 'The uniformization theorem for compact Kähler manifolds of nonnegative holomorphic bisectional curvature', J. Differential Geom. 27 (1988), no. 2, 179-214.

[35] S. Mori, 'Projective manifolds with ample tangent bundles', Ann. Math. 110(2) (1979), 593-606.

[36] L. Ni, 'Vanishing theorems on complete Kähler manifolds and their applications', J. Differential Geom. 50 (1998), no. 1, 89-122.

[37] L. Ni, 'Liouville theorems and a Schwarz Lemma for holomorphic mappings between Kähler manifolds', arXiv:1807.02674.

[38] L. Ni and F.-Y. Zheng, 'Comparison and vanishing theorems for Kähler manifolds', arXiv:1802.08732.

[39] L. Ni and F.-Y. Zheng, 'Positivity and Kodaira embedding theorem', arXiv:1804.09696.

[40] J.-C. Ottem, 'Ample subvarieties and q-ample divisors', Adv. Math. 229 (2012), no. 5, 2868-2887.

[41] T. Peternell, 'Kodaira dimension of subvarieties II', Int. J. Math. 17 (2006), 619-631.

[42] T. Peternell, 'Varieties with generically nef tangent bundles', J. Eur. Math. Soc. 14 (2012), no. 2, 571-603.

[43] Y.-T. Siu and S.-T. Yau, 'Compact Kähler manifolds of positive bisectional curvature', Invent. Math. 59(2) (1980), $189-204$.

[44] B. Totaro, 'Line bundles with partially vanishing cohomology', J. Eur. Math. Soc. 15 (2013), 731-754.

[45] B. Yang and F.-Y. Zheng, 'Hirzebruch manifolds and positive holomorphic sectional curvature', arXiv:1611.06571v2.

[46] X.-K. Yang, 'Hermitian manifolds with semi-positive holomorphic sectional curvature', Math. Res. Lett. 23 (2016), no.3, 939-952.

[47] X.-K. Yang, 'Scalar curvature on compact complex manifolds', Trans. Amer. Math. Soc. 371 (2019), no. 3, $2073-2087$.

[48] X.-K. Yang, 'A partial converse to the Andreotti-Grauert theorem', Compos. Math. 155 (2019), no. 1, 89-99.

[49] X.-K. Yang, 'RC-positivity, rational connectedness and Yau's conjecture', Camb. J. Math. 6 (2018), 183-212.

[50] X.-K. Yang, 'RC-positivity, vanishing theorems and rigidity of holomorphic maps', arXiv:1807.02601, to appear in J. Inst. Math. Jussieu.

[51] X.-K. Yang, 'RC-positivity and the generalized energy density I: Rigidity', arXiv:1810.03276.

[52] X.-K. Yang, 'Rigidity theorems on complete Kähler manifolds with RC-positive curvature', in preparation.

[53] S.-T. Yau, 'On the Ricci curvature of a compact Kähler manifold and the complex Monge-Ampère equation, I', Comm. Pure Appl. Math. 31 (1978), 339-411.

[54] S.-T. Yau, ‘A general Schwarz lemma for Kähler manifolds', Amer. J. Math.' 100 (1978), no. 1, 197-203.

[55] S.-T. Yau, problem section, 'Seminar on Differential Geometry', Ann. of Math Stud. 102 (1982), 669-706. 